\title{
Clinical Study \\ Effects of a Long-Acting Formulation of Octreotide on Patients with Portal Hypertension
}

\author{
Pei-Jing Cui, ${ }^{1}$ Jing Yao, ${ }^{2}$ Yin Zhu, ${ }^{2}$ Zheng-Yun Zhang, ${ }^{2}$ and Jun Yang ${ }^{2}$ \\ ${ }^{1}$ Department of Geriatrics, Ruijin Hospital, School of Medicine, Shanghai Jiao Tong University, Shanghai 200233, China \\ ${ }^{2}$ Department of Surgery, Shanghai Jiao Tong University Affiliated Sixth People's Hospital, Shanghai, China \\ Correspondence should be addressed to Jun Yang; yangjuns@foxmail.com
}

Received 8 February 2017; Revised 23 June 2017; Accepted 20 July 2017; Published 10 August 2017

Academic Editor: Spiros D. Ladas

Copyright (C) 2017 Pei-Jing Cui et al. This is an open access article distributed under the Creative Commons Attribution License, which permits unrestricted use, distribution, and reproduction in any medium, provided the original work is properly cited.

\begin{abstract}
Objective. This study aimed to determine whether the treatment of a long-acting formulation of octreotide (OCT-LAR) exerted a similar effect on improving the prognosis of patients with portal hypertension compared with placement of transjugular intrahepatic portosystemic shunts (TIPSs). Methods. A total of 24 patients with portal hypertension who underwent TIPS placement or OCT-LAR treatment from January 2010 to January 2015 were reviewed. Hemodynamic studies, biological values, live functions, and treatment complications before and during the treatment were evaluated. Results. Baseline clinical characteristics were similar between two groups. Hepatic venous pressure gradient (HVPG) was improved in OCT-LAR groups $(15.9 \pm 2.4$ to $12.8 \pm 1.6 \mathrm{mmHg})$. Both groups showed a slight decrease in endothelin-1 (ET-1) and urotensin II and a slight increase in oxide metabolite $(\mathrm{NO} x)$ concentrations with no significant difference. Aspartate aminotransferase and alanine aminotransferase increased one week after TIPS placement when they improved in the OCT-LAR treatment group. The complications of OCT-LAR treatment were minor and transient. However, one patient who received TIPS placement presented procedure-related complications and required rehospitalization, and 2 patients had developed hepatic encephalopathy during the follow-up period. Conclusion. Prolonged administration of OCT-LAR exerted a virtually similar effect on improving hemodynamic parameters and liver function in patients with portal hypertension compared with placement of TIPS, with no apparent serious adverse effects.
\end{abstract}

\section{Introduction}

Portal hypertension $(\mathrm{PH})$ usually results from an initial increase in intrahepatic resistance with a secondary increase in portal venous blood flow. When the pressure gradient between the portal vein and the inferior vena cava exceeds a threshold of $10 \mathrm{mmHg}$, clinical consequences including variceal bleeding, ascites, and hepatorenal syndrome become significant [1-3]. PH-related variceal bleeding remains a lifethreatening condition with a mortality rate of approximately $20 \%$. Rebleeding from the varices occurs in about $60 \%$ of patients at one to two years after the initial bleeding episode, with a mortality rate that exceeded $30 \%[4,5]$. Prevention of variceal rebleeding remains a significant challenge. Usually, the medical treatment aim is to reduce portal pressure and/or decrease blood flow in portosystemic collaterals to diminish the risk of rebleeding. Transjugular intrahepatic portosystemic shunts (TIPSs) or pharmacological therapies, such as somatostatin analogues, have usually been regarded as the preferred therapeutic strategy for this purpose. TIPS was introduced in the 1980s and has been regarded as a major technical advancement in the management of $\mathrm{PH}$ related complications [6]. The goal of TIPS placement is to reduce portal pressure by shunting blood from the portal to the systemic circulation, bypassing the liver. Currently, TIPS is overwhelmingly preferred over the traditional surgical shunts because of its less invasive technique and faster recovery time [7]. However, complications occur during or after the TIPS placement procedures, including intraperitoneal hemorrhage, portal vein perforation, and hepatic artery or bile duct injury, influencing its clinical use [8]. Octreotide (OCT), one of the somatostatin analogues, acts by inhibiting glucagon and blunting the postprandial increase in hepatic venous pressure gradient (HVPG) and portal blood flow 
[9]. Its effects in controlling variceal bleeding virtually without side effects had been proved by clinical studies and meta-analysis [10-12]. However, the current treatment regimen of subcutaneous injections three times daily is not ideal for long-term drug administration in cirrhotic patients. Hence, the introduction of OCT-LAR has made chronic treatment possible. Following a single intramuscular injection, OCT-LAR concentrations reach a plateau at day 14 and remain relatively constant for the following 3 to 4 weeks in adults [13]. A small randomized controlled study showed that after 3 months of therapy, cirrhotic patients who received $20 \mathrm{mg}$ of OCT-LAR intramuscularly every month presented a statistically significant reduction in HVPG [14]. However, the conclusion of the trial is controversial because only 18 patients were included in the trial; thus, the trial provided insufficient evidence of the efficiency profile of OCT-LAR in the cirrhotic population.

Therefore, a retrospective review of the medical records of patients who accepted TIPS placement or OCT-LAR administration after initial bleeding controlling was carried out. The purpose of this study is to decide whether the treatment of OCT-LAR exerts a similar effect in improving the prognosis with fewer complications in patients with PH compared with TIPS.

\section{Methods}

2.1. Patients. The records of patients at Shanghai Jiao Tong University Affiliated Sixth People's Hospital and Ruijing Hospital with PH who accepted TIPS placement or OCTLAR administration after initial bleeding control from January 2010 to January 2015 were reviewed. All patients with initial bleeding were treated by endoscopic band ligation at once and received systematic and prolonged use of beta blockers afterwards. A beta blocker (nonselective) was given, and the dose was adjusted to reduce baseline heart rate by $25 \%$ or to reach a pulse rate of $55 / \mathrm{min}$. 72 hours after the bleeding control, the patients were evaluated and either TIPS or LAR was given. Demographic variables, hemodynamic studies, biological values, and adverse event data before and after the treatment were collected. The diagnosis of PH was confirmed by aspiration biopsy or was made based on computed tomography (CT) and/or magnetic resonance imaging (MRI). The degree of hepatic dysfunction was graded according to the criteria of Child and Turcotte [15]. The side effects of TIPS placement or OCT-LAR during the follow-up period were recorded.

2.2. Treatment Procedure. TIPS placement was performed according to previously described methods [16] in the interventional radiology unit. After direct portography between the portal and hepatic veins, the shunt tracts were lined with various commercial stents: Wallstents (Boston Scientific Corp, Quincy, MA) and others. If necessary, balloon dilation was performed to reduce the pressure gradient. The portal systemic pressure gradient was $7-10 \mathrm{mmHg}$ in the TIPS procedure performed by our medical team.

All patients were evaluated by the same medical team according to the follow-up schedule. Doppler duplex ultrasonography (US) was performed $24 \mathrm{~h}$ and 1,3 , and 6 mo after TIPS procedure, followed by every 6 mo thereafter or whenever recurrent TIPS dysfunction was suspected clinically. TIPS dysfunction was suspected on the US if the intrastent flow velocity was less than $60 \mathrm{~cm} / \mathrm{s}$ or higher than $120 \mathrm{~cm} / \mathrm{s}$ or if there was a change in the direction of the flow in the intrahepatic portal branches compared with previous US findings. TIPS dysfunction was defined as a shunt narrowing of more than 50\%, a portosystemic pressure gradient higher than $12 \mathrm{mmHg}$ or both. Primary patency was defined as the interval of time without an intervention. A systematic invasion stent dilatation was conducted in the presence of suspected dysfunction [16].

A single dose of $20 \mathrm{mg}$ OCT-LAR (Novartis Pharma, Basel, Switzerland) was intramuscularly administrated once a month for patients who accepted pharmaceutical treatment.

2.3. Hemodynamic Studies. Hepatic hemodynamics was performed according to the recommended standard [17]. As previously described [18], an 8-F curve catheter (Cordis Europa, Amsterdam, Netherlands) was carefully introduced under fluoroscopy into the right main hepatic vein. The external zero reference was set at midchest. The wedged hepatic venous pressure (WHVP) was measured when the tip of the catheter was wedged in a small hepatic vein, providing a stable pressure tracing with fine venous fluctuations during a minimum of $30 \mathrm{~s}$. To verify that the catheter was in a proper wedged position, contrast media was also injected to visualize liver parenchyma and small portal branches. The free hepatic venous pressure (FHVP) was measured when the catheter floated in the hepatic vein near the junction with the vena cava. HVPG, which is an estimate of portal pressure [19], results from the difference between the WHVP and FHVP.

2.4. Assessment of Biological Values. The hepatic venous blood was sampled during hepatic hemodynamic studies. Samples taken in heparinized tubes were transported immediately on ice to the laboratory. After centrifugation for $15 \mathrm{~min}$ at $5^{\circ} \mathrm{C}$, aliquots were prepared under pyrogen-free conditions and kept frozen at $-70^{\circ} \mathrm{C}$ until assay. The serum concentrations of ET-1 and urotensin II (URO II) were measured by commercially available enzyme-linked immunosorbent assay (ELISA) kits according to the manufacturer's guidelines. The plasma concentration of oxide metabolites (nitrite and nitrate as $\mathrm{NO} x$ ) was measured using a specific fluorometric assay (Cayman Chemical Company, Ann Arbor, MI).

Peripheral blood samples were analyzed for aspartate aminotransferase (AST), alanine aminotransferase (ALT), total bilirubin (TB), and prothrombin time (PT) by using standard laboratory methods.

2.5. Statistics. Results are expressed as mean and standard error of the mean (SEM), and all statistical analyses were performed using the Statview 5.0 Program (Abacus Concepts, Berkeley, CA). A $P$ value of less than 0.05 was considered statistically significant. 
TABle 1: Patient characteristics.

\begin{tabular}{lccc}
\hline & TIPS & OCT-LAR & $P$ \\
\hline Patients (M/F) & $9 / 3$ & $8 / 4$ & 0.65 \\
Age (years) & $49.6 \pm 8.7$ & $50.8 \pm 9.8$ & 0.76 \\
Child scores & $8.1 \pm 0.5$ & $8.0 \pm 0.6$ & 0.72 \\
Chronic active & $9 / 3$ & $9 / 3$ & 1.0 \\
hepatitis/alcoholic cirrhosis & & & \\
Follow-up time (d) & $79.2 \pm 19.9$ & $82.1 \pm 16.8$ & 0.69 \\
\hline
\end{tabular}

TABLE 2: Hemodynamic values at baseline and last follow-up.

\begin{tabular}{lccc}
\hline OCT-LAR $(N=12)$ & Baseline & Last follow-up & $P$ \\
\hline WHVP $(\mathrm{mmHg})$ & $26.9 \pm 2.3$ & $22.6 \pm 1.6$ & $<0.05$ \\
FHVP $(\mathrm{mmHg})$ & $11.0 \pm 1.1$ & $9.8 \pm 1.3$ & $<0.05$ \\
HVPG $(\mathrm{mmHg})$ & $15.9 \pm 2.4$ & $12.8 \pm 1.6$ & $<0.05$ \\
\hline
\end{tabular}

\section{Results}

3.1. Characteristics of Patients. The two groups of patients were well matched in sex, age, severity of liver dysfunction, cause of liver disease, and follow-up time (Table 1).

3.2. Hemodynamic Studies. Hemodynamic studies were successfully performed in the OCT-LAR groups at baseline and at the end of follow-up. Results are presented in Table 2. At the end of the follow-up, HVPG was reduced in the OCT-LAR group $(15.9 \pm 2.4$ to $12.8 \pm 1.6 \mathrm{mmHg}$, $P<0.05)$.

3.3. Biological Values. The evolution of biological tests over the follow-up period is given in Table 3. No statistical difference was found in the baseline plasma concentrations of ET- 1 and URO II between the TIPS and OCT-LAR groups $(5.9 \pm 0.5 \mathrm{pg} / \mathrm{mL}$ versus $6.1 \pm 0.3 \mathrm{pg} / \mathrm{mL}, P=0.25 ; 2399.9 \pm$ $107.8 \mathrm{pg} / \mathrm{mL}$ versus $2453.3 \pm 77.8 \mathrm{pg} / \mathrm{mL}, P=0.18$, resp.). A slight decrease in ET-1 and URO II was observed in both groups at the end of follow-up but without statistical difference between them $(5.6 \pm 0.4$ versus $5.7 \pm 0.3 \mathrm{pg} / \mathrm{mL}$, $P=0.41 ; 2344.8 \pm 91.9$ versus $2419.3 \pm 103.8 \mathrm{pg} / \mathrm{mL}, P=0.08$, resp.). NO $x$ concentrations remained statistically unchanged in the TIPS and OCT-LAR groups both at baseline and last follow-up $(64.9 \pm 2.7$ versus $65.5 \pm 2.6 \mu \mathrm{mol} / \mathrm{L}, P=0.62$; $67.9 \pm 2.6$ versus $68.5 \pm 2.0 \mu \mathrm{mol} / \mathrm{L}, P=0.5$, resp.).

3.4. Live Functions. No statistical difference in baseline liver function was observed between the two groups (Table 4). An acute elevation of hepatobiliary enzymes, including AST and ALT at one week after the TIPS placement, was observed. At the same time point, the two hepatobiliary enzymes were improved in the OCT-LAR treatment group. However, no significant difference was found between the groups (Table 5). Two groups of patients did not show different improvement in liver function, assessed by plasma concentrations of AST, ALT, TB, and PT at the end of follow-up (Table 5).
TABLE 3: Biological values at baseline and last follow-up.

\begin{tabular}{lccc}
\hline & TIPS $(N=12)$ & OCT-LAR $(N=12)$ & $P$ value \\
\hline Baseline & & & \\
ET-1 $(\mathrm{pg} / \mathrm{mL})$ & $5.9 \pm 0.5$ & $6.1 \pm 0.3$ & 0.25 \\
URO II $(\mathrm{pg} / \mathrm{mL})$ & $2399.9 \pm 107.8$ & $2453.3 \pm 77.8$ & 0.18 \\
NOx $(\mu \mathrm{mol} / \mathrm{L})$ & $64.9 \pm 2.7$ & $65.5 \pm 2.6$ & 0.62 \\
Last follow-up & & & \\
ET-1 $(\mathrm{pg} / \mathrm{mL})$ & $5.6 \pm 0.4$ & $5.7 \pm 0.3$ & 0.41 \\
URO II $(\mathrm{pg} / \mathrm{mL})$ & $2344.8 \pm 91.9$ & $2419.3 \pm 103.8$ & 0.08 \\
NO $x(\mu \mathrm{mol} / \mathrm{L})$ & $67.9 \pm 2.6$ & $68.5 \pm 2.0$ & 0.50 \\
\hline
\end{tabular}

TABLE 4: Live functions at baseline.

\begin{tabular}{lccc}
\hline & TIPS $(N=12)$ & OCT-LAR $(N=12)$ & $P$ \\
\hline AST $(\mathrm{U} / \mathrm{L})$ & $94.6 \pm 49.3$ & $97.2 \pm 37.3$ & 0.89 \\
ALT $(\mathrm{U} / \mathrm{L})$ & $101.3 \pm 57.2$ & $100.8 \pm 35.3$ & 0.98 \\
TB $(\mu \mathrm{mol} / \mathrm{L})$ & $64.7 \pm 16.9$ & $51.9 \pm 16.3$ & 0.07 \\
Prothrombin time $(\mathrm{s})$ & $17.6 \pm 1.3$ & $18.1 \pm 1.8$ & 0.51 \\
\hline
\end{tabular}

TABle 5: Live functions at one week and last follow-up.

\begin{tabular}{lccc}
\hline & TIPS $(N=12)$ & OCT-LAR $(N=12)$ & $P$ \\
\hline One week & & & \\
AST (U/L) & $101.8 \pm 49.2$ & $90.1 \pm 40.6$ & 0.53 \\
ALT (U/L) & $104.8 \pm 48.1$ & $99.6 \pm 50.6$ & 0.80 \\
TB ( $\mu$ mol/L) & $53.4 \pm 11.2$ & $48.6 \pm 11.6$ & 0.34 \\
PT (s) & $17.5 \pm 1.3$ & $17.8 \pm 2.1$ & 0.63 \\
Last follow-up & & & \\
AST (U/L) & $85.8 \pm 44.6$ & $81.8 \pm 41.6$ & 0.82 \\
ALT (U/L) & $91.8 \pm 49.5$ & $80.3 \pm 38.2$ & 0.53 \\
TB $(\mu \mathrm{mol} / \mathrm{L})$ & $45.4 \pm 11.7$ & $42.1 \pm 9.7$ & 0.47 \\
PT $(\mathrm{s})$ & $16.1 \pm 1.0$ & $17.0 \pm 1.7$ & 0.13 \\
\hline
\end{tabular}

3.5. Treatment Complications. One patient in every group presented minor gastrointestinal bleeding (not life threatening and only melena is manifested which means possible rebleeding) during the follow-up period. The complications of OCT-LAR treatment were minor, consisting of abdominal cramps (two patients), transient diarrhea (three patients), hypoglycemia (one patient), flatulence (two patients), and dizziness (two patients). No patient with OCT-LAR treatment developed gallstones, biliary colic, hypertension, or hepatic encephalopathy. However, one patient who received TIPS placement presented procedure-related complications and required rehospitalization during the follow-up period, and 2 patients who received TIPS placement had developed hepatic encephalopathy (Table 6).

\section{Discussions}

Variceal hemorrhage is the most serious complication of $\mathrm{PH}$, accounting for $17-57 \%$ of all deaths in cirrhotic patients [20]. The main therapeutic goal in patients with $\mathrm{PH}$ is the 
TABLE 6: Treatment complications.

\begin{tabular}{lcc}
\hline & TIPS $(N=12)$ & OCT-LAR $(N=12)$ \\
\hline Rebleeding & 1 & 1 \\
Deaths & 0 & 0 \\
Serious* & 1 & 0 \\
Abdominal cramps & 0 & 2 \\
Diarrhea & 0 & 3 \\
Hypoglycemia & 0 & 1 \\
Constipation & 0 & 0 \\
Flatulence & 0 & 2 \\
Cholelithiasis/biliary colic & 0 & 0 \\
Hypertension & 0 & 0 \\
Dizziness & 0 & 2 \\
Hepatic encephalopathy & 2 & 0 \\
\hline
\end{tabular}

*Serious means requiring hospitalization, fatally life threatening, significantly incapacitating, or requiring medical intervention.

prevention of initial and recurrent variceal bleeding. Over the past two decades, numerous advances in medical and procedural treatments have been developed for this purpose. One of the largest changes has been the development of TIPS. At present, TIPS placement is generally considered as a first-line therapy in the definitive treatment of $\mathrm{PH}$ concomitant with variceal bleeding [21] or as a bridge to liver transplantation because of its relatively low cost and an easier transplantation process [22]. A study showed that TIPS placement within $72 \mathrm{~h}$ after acute bleeding can effectively prevent recurrent variceal bleeding [23]. However, complications occur during or after TIPS placement procedures, influencing its clinical use. Therefore, pharmacological agents that may help to improve the prognosis of patients by successfully controlling the initial variceal bleeding could represent an interesting option for those patients who are not candidates for TIPS.

Somatostatin analogues, such as OCT, remain a good choice for acute variceal hemorrhage episodes in patients with $\mathrm{PH}[24,25]$ and also proved to be an effective method to prevent recurrent bleeding episodes [26] with well-known mechanisms for lowering portal pressure [27]. However, the requirement of three times daily administration renders the method impractical for longterm prophylaxis. The long-acting formulation of OCT, namely, OCT-LAR, can be administered once per month with similar efficacy and safety profile to subcutaneous daily administration [28]. Contraction of the vascular smooth muscle via somatostatin receptors and effects on neurohumoral systems on splanchnic vasculature may explain the hemodynamic changes associated with OCTLAR [29]. After a single intramuscular injection, concentrations of OCT-LAR reach a plateau at day 14 and remain relatively constant for the following 3 to 4 weeks [30]. Therefore, the approach may represent an attractive option for the long-term therapy of variceal bleeding. Spahr et al. [14] suggested that the prolonged administration of OCT-LAR significantly improves the prognosis in several cirrhosis patients.
The purpose of our study is to decide whether the treatment of OCT-LAR exerts a similar effect in improving the prognosis in patients with $\mathrm{PH}$ compared with TIPS. Indeed, the choice of TIPS procedure or OCT-LAR administration was based on a clinician's decision only. All patients presented similar characteristics at baseline, were in a stable clinical condition, and remained so during a mean followup of around 80 days regardless of whether belonging to the TIPS or OCT-LAR groups.

We use HVPG as a reliable indicator of portal pressure in our study, as supported by numerous authors [31-33]. We also make it one of the most important indicators to judge the effectiveness of OCT-LAR treatment. HVPG had been measured at baseline and at last follow-up time. Our results showed that mean HVPG decreased from $15.9 \pm 2.4$ to $12.8 \pm 1.6 \mathrm{mmHg}$ after the administration of OCT-LAR, which is a significant improvement with about $20 \%$ reduction. We also showed that reduction in HVPG by OCTLAR precedes an improvement in liver function. We found a decrease in AST, ALT, TB, and prothrombin time after 1 week of OCT-LAR administration. The mechanism by which OCT-LAR stimulates liver function remains unclear but could be due to direct or indirect effects on hepatocytes [34]. On the contrary, TIPS resulted in a transient elevation of hepatobiliary enzymes, especially at an early stage after the procedure with an acute increase in AST and ALT in our study. The transient nature of these alterations may be explained by inevitable liver injury both by direct mechanical insult to the hepatic parenchyma as well as potentially decreased antegrade intrahepatic portal venous flow, arterial perfusional compensation, and resultant perfusional ischemia. It is known that a decrease in portal venous blood flow results in an increase in hepatic arterial flow in a mechanism termed the "hepatic arterial buffer response" [35], a phenomenon that may begin instantly after TIPS placement [36]. Although, the temporal evolution of liver function parameters is decreased at the end of follow-up, the liver injury may have an exaggerated effect on patients with poor liver function, which put them at an irreversible higher risk of early mortality [37].

Intrahepatic vascular resistance is regulated by the balance between vasoactive agents, such as ET-1, and vasorelaxing agents, such as nitric oxide (NO). In a cirrhotic liver, increased levels of ET-1 and decreased levels of NO contribute to the contraction of hepatic stellate cells [38]. Wereszczynka-Siemiatkowska et al. showed that peripheral ET-1 levels reflect the stage of $\mathrm{PH}$ and liver insufficiency in cirrhosis [39] and are correlated significantly with liver laboratory parameters and HVPG. In our study, we found that after TIPS placement and OCT-LAR treatment, a slight decrease in ET-1 and an increase in NOx levels in hepatic venous blood were observed during follow-up period but without difference. Vascular endothelial cells in an enlarged spleen may be an important source of ET-1; OCT-LAR exerted a similar effect on reducing portal pressure and improved the enlarged spleen compared with TIPS. The increase in NOx level may be explained by restoring production when subsequent improvements in sinusoidal circulation may increase shear stress on sinusoidal endothelial 
cells. Moreover, we also found the same downtrend of URO II. As somatostatin-like peptide, URO II has recently been recognized as the most potent vascular mediator in humans, being a minimum of an order of magnitude more potent than ET-1 [40]. Kemp et al. [41] showed that serum URO II is elevated in patients with chronic liver disease and is associated with the severity of the underlying liver disease and the degree of $\mathrm{PH}$. Together with the results of ET-1, NOx, and URO II, OCT-LAR and TIPS placement are suggested to exert a possible similar effect in improving the prognosis of $\mathrm{PH}$.

In our study, treatment complications related with OCTLAR are similar with that in a recent article aimed at investigating the safety and efficacy of OCT-LAR in patients with $\mathrm{PH}$. The study only reported abdominal cramps, diarrhea, and hypoglycemia, whereas no serious adverse effects were detected during the treatment period [42].

The data provided in this study showed similar incidence of minor bleeding and similar liver function, but more clinical complications in the TIPS group. TIPS is a traumatic procedure, and we intended to find some clues in this study to support our notion that LAR could substitute TIPS in some extend. Though they had a similar incidence of minor bleeding and similar liver function, LAR had less clinical complications than TIPS. Therefore, LAR is preferred in our point of view when a treatment decision is made.

In conclusion, our data showed that the prolonged administration of OCT-LAR presented a virtually similar effect on improving the hemodynamic parameters and liver function in patients with $\mathrm{PH}$ compared with TIPS, with no apparent serious adverse effects. The approach is effective in reducing the possibility of $\mathrm{PH}$-related recurrent variceal bleeding in patients with advanced $\mathrm{PH}$. However, owing to the retrospective nature of the approach, further studies on long-term treatment with OCT-LAR in larger groups of patients are still needed.

\section{Conflicts of Interest}

None of the authors have any commercial or other association that might pose a conflict of interests.

\section{Authors' Contributions}

Pei-Jing Cui and Jing Yao contributed equally to this work.

\section{Acknowledgments}

This work was supported by the grant from the National Natural Science Foundation of China (81501086).

\section{References}

[1] G. Garcia-Tsao, A. J. Sanyal, N. D. Grace, W. Carey, and Practice Guidelines Committee of the American Association for the Study of Liver Diseases; Practice Parameters Committee of the American College of Gastroenterology, "Prevention and management of gastroesophageal varices and variceal hemorrhage in cirrhosis," Hepatology, vol. 46, no. 3, pp. 922938, 2007.
[2] A. J. Sanyal, J. Bosch, A. Blei, and V. Arroyo, "Portal hypertension and its complications," Gastroenterology, vol. 134, no. 6, pp. 1715-1728, 2008.

[3] J. O’Brien, C. Triantos, and A. K. Burroughs, "Management of varices in patients with cirrhosis," Nature Reviews Gastroenterology \& Hepatology, vol. 10, no. 7, pp. 402-412, 2013.

[4] D. Y. Graham and J. L. Smith, "The course of patients after variceal hemorrhage," Gastroenterology, vol. 80, no. 4, pp. 800-809, 1981.

[5] J. L. Smith and D. Y. Graham, "Variceal hemorrhage: a critical evaluation of survival analysis," Gastroenterology, vol. 82, no. 5, Part 1, pp. 968-973, 1982.

[6] R. F. Colapinto, R. D. Stronell, M. Gildiner et al., "Formation of intrahepatic portosystemic shunts using a balloon dilatation catheter: preliminary clinical experience," $A J R$ American Journal of Roentgenology, vol. 140, no. 4, pp. 709$714,1983$.

[7] S. Siramolpiwat, "Transjugular intrahepatic portosystemic shunts and portal hypertension-related complications," World Journal of Gastroenterology, vol. 20, no. 45, pp. 16996-17010, 2014.

[8] R. Ripamonti, H. Ferral, M. Alonzo, and N. H. Patel, "Transjugular intrahepatic portosystemic shunt-related complications and practical solutions," Seminars in Interventional Radiology, vol. 23, no. 2, pp. 165-176, 2006.

[9] R. Jalan, P. C. Hayes, and British Society of Gastroenterology, "UK guidelines on the management of variceal haemorrhage in cirrhotic patients," Gut, vol. 46, Supplement 3-4, pp. III1IIII $15,2000$.

[10] P. A. McCormick, M. R. Biagini, R. Dick et al., "Octreotide inhibits the meal-induced increases in the portal venous pressure of cirrhotic patients with portal hypertension: a doubleblind, placebo-controlled study," Hepatology, vol. 16, no. 5, pp. 1180-1186, 1992.

[11] A. Albillos, I. Rossi, J. Iborra et al., "Octreotide prevents postprandial splanchnic hyperemia in patients with portal hypertension," Journal of Hepatology, vol. 21, no. 1, pp. 8894, 1994.

[12] D. A. Corley, J. P. Cello, W. Adkisson, W. F. Ko, and K. Kerlikowske, "Octreotide for acute esophageal variceal bleeding: a meta-analysis," Gastroenterology, vol. 120, no. 4, pp. 946-954, 2001

[13] M. O'Meara, M. P. Cicalese, A. Bordugo, A. Ambrosi, N. Hadzic, and G. Mieli-Vergani, "Successful use of long-acting octreotide for intractable chronic gastrointestinal bleeding in children," Journal of Pediatric Gastroenterology and Nutrition, vol. 60, no. 1, pp. 48-53, 2015.

[14] L. Spahr, E. Giostra, J. L. Frossard, I. Morard, G. Mentha, and A. Hadengue, "A 3-month course of long-acting repeatable octreotide (Sandostatin LAR) improves portal hypertension in patients with cirrhosis: a randomized controlled study," The American Journal of Gastroenterology, vol. 102, no. 7, pp. 1397-1405, 2007.

[15] C. G. Child and J. G. Turcotte, "Surgery and portal hypertension," Major Problems in Clinical Surgery, vol. 1, pp. 1-85, 1964.

[16] R. C. Gaba, B. O. Omene, E. S. Podczerwinski et al., "TIPS for treatment of variceal hemorrhage: clinical outcomes in 128 patients at a single institution over a 12-year period," Journal of Vascular and Interventional Radiology, vol. 23, no. 2, pp. 227-235, 2012. 
[17] R. J. Groszmann and S. Wongcharatrawee, "The hepatic venous pressure gradient: anything worth doing should be done right," Hepatology, vol. 39, no. 2, pp. 280-282, 2004.

[18] L. Spahr, E. Giostra, I. Morard, G. Mentha, and A. Hadengue, "Perendoscopic variceal pressure measurement: a reliable estimation of portal pressure in patients with cirrhosis?," Gastroentérologie Clinique et Biologique, vol. 30, no. 8-9, pp. 1012-1018, 2006.

[19] P. M. Huet and G. Pomier-Layrargues, "The hepatic venous pressure gradient: "remixed and revisited"," Hepatology, vol. 39, no. 2, pp. 295-298, 2004.

[20] R. de Franchis, "Revising consensus in portal hypertension: report of the Baveno $\mathrm{V}$ consensus workshop on methodology of diagnosis and therapy in portal hypertension," Journal of Hepatology, vol. 53, no. 4, pp. 762-768, 2010.

[21] W. S. Helton, R. Maves, K. Wicks, and K. Johansen, “Transjugular intrahepatic portosystemic shunt vs surgical shunt in good-risk cirrhotic patients: a case-control comparison," Archives of Surgery, vol. 136, no. 1, pp. 17-20, 2001.

[22] A. R. Bonnel, C. Bunchorntavakul, and R. K. Rajender, "Transjugular intrahepatic portosystemic shunts in liver transplant recipients," Liver Transplantation, vol. 20, no. 2, pp. 130139, 2014.

[23] J. C. Garcia-Pagan, K. Caca, C. Bureau et al., "Early use of TIPS in patients with cirrhosis and variceal bleeding," The New England Journal of Medicine, vol. 362, no. 25, pp. 2370-2379, 2010.

[24] J. J. Sung, S. C. Chung, C. W. Lai et al., "Octreotide infusion or emergency sclerotherapy for variceal haemorrhage," Lancet, vol. 342, no. 8872, pp. 637-641, 1993.

[25] M. Gugger, B. Waser, A. Kappeler, A. Schonbrunn, and J. C. Reubi, "Cellular detection of sst2A receptors in human gastrointestinal tissue," Gut, vol. 53, no. 10, pp. 1431-1436, 2004.

[26] S. A. Jenkins, J. N. Baxter, M. Critchley et al., "Randomised trial of octreotide for long term management of cirrhosis after variceal haemorrhage," British Medical Journal, vol. 315, no. 7119, pp. 1338-1341, 1997.

[27] R. Chatila, L. Ferayorni, T. Gupta, and R. J. Groszmann, "Local arterial vasoconstriction induced by octreotide in patients with cirrhosis," Hepatology, vol. 31, no. 3, pp. 572-576, 2000.

[28] L. B. Anthony, "Long-acting formulations of somatostatin analogues," Italian Journal of Gastroenterology and Hepatology, vol. 31, Supplement 2, pp. S216-S218, 1999.

[29] A. J. Sanyal, "Octreotide and its effects on portal circulation," Gastroenterology, vol. 120, no. 1, pp. 303-305, 2001.

[30] J. Bornschein, I. Drozdov, and P. Malfertheiner, "Octreotide LAR: safety and tolerability issues," Expert Opinion on Drug Safety, vol. 8, no. 6, pp. 755-768, 2009.

[31] A. Armonis, D. Patch, and A. Burroughs, "Hepatic venous pressure measurement: an old test as a new prognostic marker in cirrhosis?" Hepatology, vol. 25, no. 1, pp. 245-248, 1997.

[32] C. Villanueva, J. M. Lopez-Balaguer, C. Aracil et al., "Maintenance of hemodynamic response to treatment for portal hypertension and influence on complications of cirrhosis," Journal of Hepatology, vol. 40, no. 5, pp. 757-765, 2004.

[33] C. Ripoll, R. J. Groszmann, G. Garcia-Tsao et al., "Hepatic venous pressure gradient predicts development of hepatocellular carcinoma independently of severity of cirrhosis," Journal of Hepatology, vol. 50, no. 5, pp. 923-928, 2009.

[34] I. Landa, J. Arias, M. Gomez, M. Quadros, A. Moreno, and J. L. Balibrea, "Cytoprotective effect of somatostatin in a rat model of hepatic ischemic reperfusion," Hepatology, vol. 16, no. 6, pp. 1474-1476, 1992.

[35] A. Zipprich, "Hemodynamics in the isolated cirrhotic liver," Journal of Clinical Gastroenterology, vol. 41, Supplement 3, pp. S254-S258, 2007.

[36] B. Radeleff, C. M. Sommer, T. Heye et al., "Acute increase in hepatic arterial flow during TIPS identified by intravascular flow measurements," Cardiovascular and Interventional Radiology, vol. 32, no. 1, pp. 32-37, 2009.

[37] L. C. Casadaban, A. Parvinian, P. M. Couture et al., "Characterization of liver function parameter alterations after transjugular intrahepatic portosystemic shunt creation and association with early mortality," AJR American Journal of Roentgenology, vol. 203, no. 6, pp. 1363-1370, 2014.

[38] D. Rockey, "The cellular pathogenesis of portal hypertension: stellate cell contractility, endothelin, and nitric oxide," Нераtology, vol. 25, no. 1, pp. 2-5, 1997.

[39] U. Wereszczynka-Siemiatkowska, A. Swidnicka-Siergiejko, A. Siemiatkowski et al., "Endothelin 1 and transforming growth factor-betal correlate with liver function and portal pressure in cirrhotic patients," Cytokine, vol. 76, no. 2, pp. 144-151, 2015.

[40] R. S. Ames, H. M. Sarau, J. K. Chambers et al., "Human urotensin-II is a potent vasoconstrictor and agonist for the orphan receptor GPR14," Nature, vol. 401, no. 6750, pp. 282-286, 1999.

[41] W. Kemp, H. Krum, J. Colman et al., "Urotensin II: a novel vasoactive mediator linked to chronic liver disease and portal hypertension," Liver International, vol. 27, no. 9, pp. 1232-1239, 2007.

[42] N. Chandok, P. S. Kamath, A. Blei et al., "Randomised clinical trial: the safety and efficacy of long-acting octreotide in patients with portal hypertension," Alimentary Pharmacology \& Therapeutics, vol. 35, no. 8, pp. 904-912, 2012. 


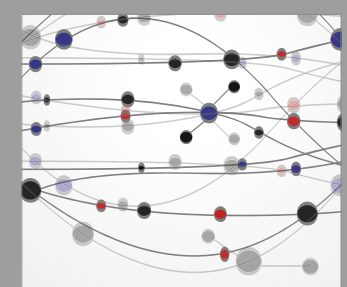

The Scientific World Journal
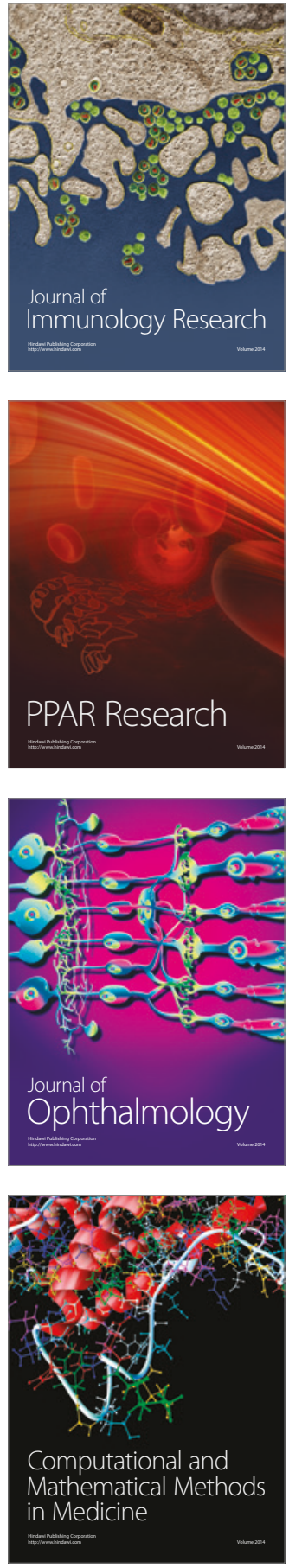

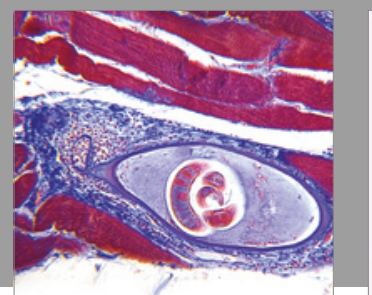

Gastroenterology Research and Practice
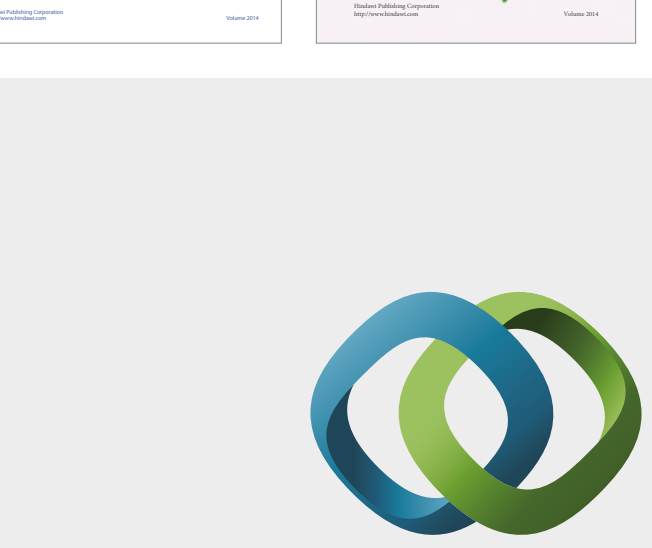

\section{Hindawi}

Submit your manuscripts at

https://www.hindawi.com
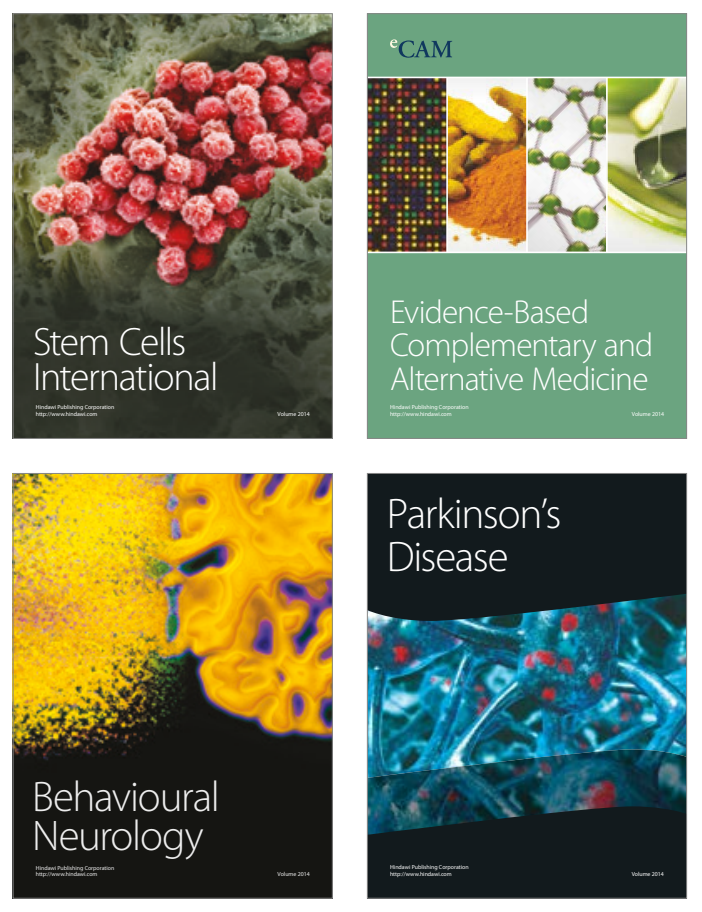
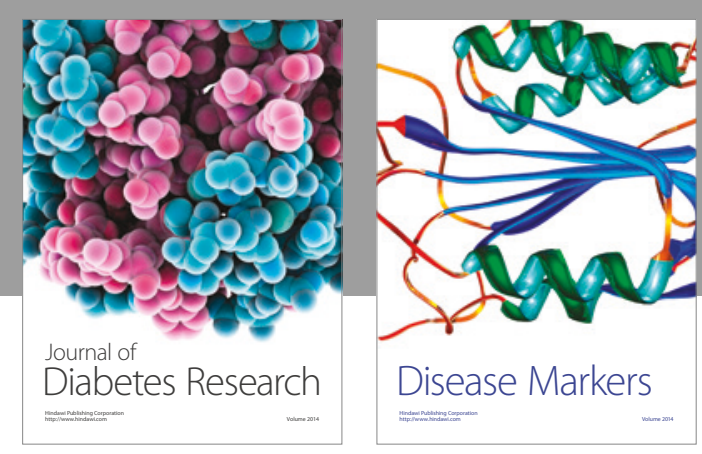

Disease Markers
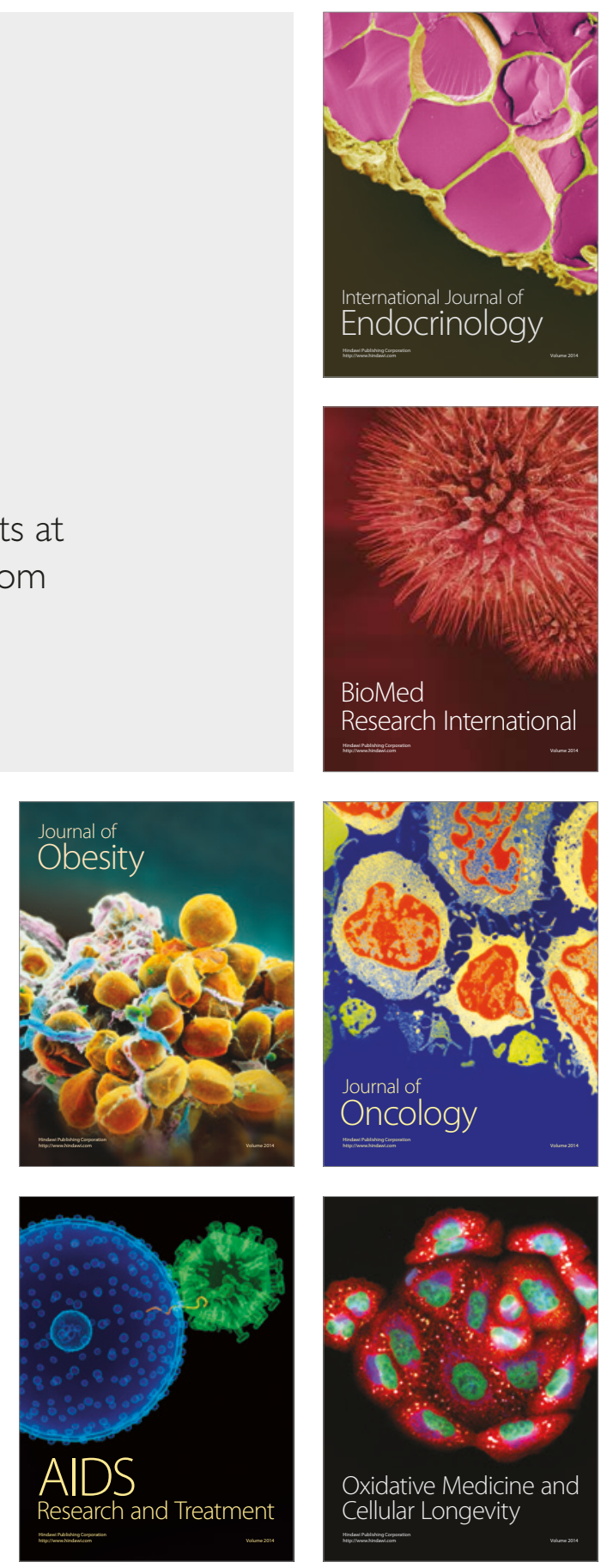